\title{
Appraisal of the total blood lymphocyte proliferation assay as a diagnostic tool in screening for tuberculosis
}

\author{
N. P. FIAVEY* and S. FRANKENBURG $\dagger$
}

School of Public Health, Hebrew University, Hadassah Medical School, Jerusalem 91010 and $\dagger$ Department of Dermatology, Hadassah University Hospital and Department of Parasitology. The Kuvin Center for Research into Tropical and Infectious Diseases, PO Box 12000, Jerusalem 91120, Israel

\begin{abstract}
Summary. The total blood lymphocyte proliferation assay (TLP) was evaluated as a screening test for infection with Mycobacterium tuberculosis and was compared with the tuberculin (Mantoux) skin test. The results of TLP assays performed on 33 patients with tuberculosis and 37 non-tuberculous subjects were compared with results of skin tests performed in the previous year. There was a high correlation between skin test responses and TLP responses to PPD which was statistically significant. The sensitivity, specificity and the predictive value of a positive test were also similar for the skin test and TLP test. These findings suggest that the TLP test is as effective in screening for $M$. tuberculosis infection as tuberculin skin testing. Future research leading to further simplification of the TLP method may lead to it replacing intradermal skin testing.
\end{abstract}

\section{Introduction}

Although the aetiological agent of human tuberculosis was discovered by Robert Koch more than 100 years ago, early detection of infection with $\mathrm{Myco}$ bacterium tuberculosis is still difficult in spite of the tremendous advances in the study of this disease. Infection by the organism is closely associated with delayed hypersensitivity (DH) to tuberculin, perhaps the best recognised example of a cell-mediated immune response in man. ${ }^{1}$ Originally, the response to $M$. tuberculosis was studied in the tuberculin skin test. However, during the past 25 years, several in-vitro techniques have become available, some of which have been found to be equally sensitive indicators of tuberculous infection. ${ }^{2,3}$ Proliferation of peripheral blood lymphocytes in vitro in response to antigens of $M$. tuberculosis is generally correlated with the skin test response to PPD, although instances have been reported of lymphocyte proliferation in the absence of a DH response to PPD. ${ }^{4}$

Lymphocyte proliferation in vitro is an accepted technique for assessing cell-mediated immunity (CMI). However, because it requires quantities of blood that can be obtained only by venepuncture, and because of the need to separate the mononuclear cells, the technique has been too cumbersome for routine use. Recently, we have developed a modification of the

Received 19 Sept. 1991; accepted 7 Jan. 1992.

* Present address and address for correspondence: Department of Microbiology and Immunology, (Mail Stop 410), Hahnemann University School of Medicine, Broad and Vine, Philadelphia, PA 19102-1192, USA. lymphocyte proliferation assay, which can be performed on 100- $\mu$ l samples of whole blood, a quantity that can be obtained by fingerprick. In a study of individuals exposed to infection by Leishmania major or vaccinated with BCG, the simplified assay yielded results comparable to those of the classical assay employing separated peripheral blood mononuclear cells. $^{5}$

The objective of the present study was to determine the significance of the modified whole-blood lymphocyte proliferation assay in subjects with or without tuberculosis and to evaluate this method of screening for infection by $M$. tuberculosis.

\section{Materials and methods}

\section{Subjects}

Seventy patients attending two centres for chest diseases in Israel, were recruited; 33 were patients with tuberculosis who met the criteria of the Israeli Ministry of Health-i.e., clinical and radiological evidence, together with bacteriological confirmation-and 37 were patients with chest diseases other than tuberculosis. None of the subjects had a known history of BCG vaccination. In both groups, the age range was 21-93 years (median 62 years) and the numbers of males and females were approximately equal.

\section{Skin tests}

The results of tuberculin skin tests performed during the previous year were obtained from the patients' 
records; in most cases these had been recorded simply as "positive" or "negative". In those cases in which the diameter of the skin test responses had been recorded, tests yielding diameters $\geqslant 10 \mathrm{~mm}$ were considered to be positive.

\section{Total blood lymphocyte proliferation assay}

The total blood lymphocyte proliferation assay (TLP) was performed as described previously. ${ }^{5}$ Briefly, $100-\mu$ l samples of blood obtained by fingerprick were drawn into sterile, disposable $100-\mu 1$ micropipettes (Blaubrand, intraMark, Germany), and transferred into tubes containing $1.5 \mathrm{ml}$ of RPMI 1640 cell-culture medium (Ysum, Israel) supplemented with glutamine $2 \mathrm{mmol}$, streptomycin $100 \mathrm{mg} / \mathrm{L}$, penicillin $10^{5} \mathrm{U} / \mathrm{L}$ (Teva, Israel) and $1 \mu \mathrm{l}$ of heparin without preservatives (Leo, Ballerup, Denmark). The diluted blood was mixed thoroughly and distributed in $100-\mu \mathrm{l}$ volumes into round-bottomed 96-well plates (Cel-cult, Sterlin). Mitogen (Phytohaemagglutinin-M, PHA; Difco) $0.3 \mu \mathrm{g} /$ well and antigen (Tuberculin Purified Protein Derivative, PPD; Statens Seruminstitut, Copenhagen, Denmark) 1 or $8 \mu \mathrm{g} /$ well were added in $25-\mu \mathrm{l}$ volumes. The plates were incubated for 6 days at $37^{\circ} \mathrm{C}$ in an atmosphere of air plus $\mathrm{CO}_{2} 6 \% ; 6 \mathrm{~h}$ before harvesting, $1 \mu \mathrm{Ci}$ of ${ }^{3} \mathrm{H}$-thymidine in a volume of $25 \mu \mathrm{l}$ (Nuclear Research Center, Israel; $2 \mathrm{Ci} / \mathrm{mmol}$ ) was added to each well. Every test was performed in triplicate. Cells were harvested with an automatic cellharvester (Titertek), and the incorporated ${ }^{3} \mathrm{H}$ thymidine was assayed by liquid scintillation spectrometry (Packard Tricarb). Results are presented as counts/min (cpm), or as stimulation index:

$\mathrm{cpm}$ of antigen containing wells/cpm of wells containing cells without antigen.

\section{Statistical analysis}

Results of the skin test and the TLP test were analysed statistically by means of $\chi^{2}$-test and Fisher's test of exact probability. Correlation was determined in terms of the strength of association between pairs of variables by calculating Odds ratios, because of the qualitative nature of the skin test results.

\section{Results}

Cells were considered to have responded to PPD in the TLP assay if, in the presence of one of the concentrations of the antigen, the $\mathrm{cpm} /$ were $\geqslant 3000$ and the stimulation index $\geqslant 3 .{ }^{5}$ In this study, all the specimens that yielded $\mathrm{cpm} \geqslant 3000$ also yielded a stimulation index $\geqslant 3$, therefore only the results of cpm values are presented.

Of the 33 subjects whose cells responded to PPD, $70 \%$ demonstrated higher responses to $8 \mu \mathrm{g}$ (average cpm 11923 SD 8058) and $30 \%$ higher responses to $1 \mu \mathrm{g}$ PPD (average cpm 14392 SD 10583). The higher response was taken as that subject's response to PPD.

The responses to PPD in the TLP and the skin test are shown in the figure. These data demonstrate that $75 \%$ of those failing to react to PPD in the TLP also failed to respond in the skin test. Similarly, c. $75 \%$ of those responding in the TLP had also given a positive response to PPD in the skin test. The Odds ratios for the association of responses in the TLP and the skin test were 8.5 among the tuberculosis patients, and 12.0 among the controls $(\mathrm{p}<0.01)$. Thus, there appeared

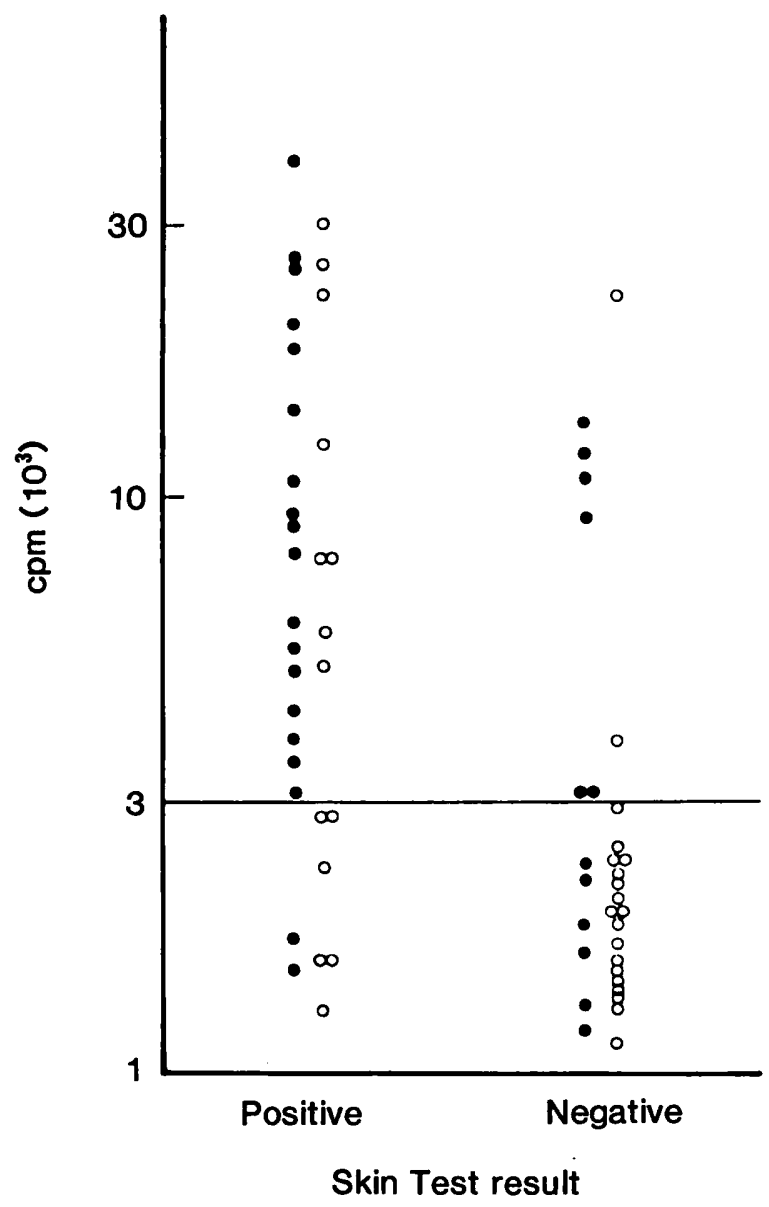

Figure. Comparison of response to PPD in the TLP assay (cpm) and in the skin test; $O$ cases, $O$ controls. For each individual, the higher cpm values are shown.

Table. Comparison of skin test and TLP test with the diagnosis of tuberculosis by the physicians

\begin{tabular}{|c|c|c|c|c|}
\hline & \multicolumn{3}{|c|}{ Number of subjects } & \\
\hline & Cases & Controls & Total & \\
\hline \multicolumn{5}{|c|}{ Skin test result } \\
\hline Positive & 19 & 14 & $33)$ & Sensitivity $61.3 \%$ \\
\hline Negative & 12 & 20 & 32 & $\begin{array}{l}\text { Predictive value of a } \\
\text { positive test } 57.6 \% \text {. }\end{array}$ \\
\hline Total & 31 & 34 & 65 & Specificity $58.8 \%$. \\
\hline \multicolumn{5}{|l|}{ TLP test } \\
\hline Positive & 21 & 15 & $36)$ & Sensitivity $63.6 \%$. \\
\hline Negative & 12 & 22 & 34 & $\begin{array}{l}\text { Predictive value of a } \\
\text { positive test } 58.3 \% \text {. }\end{array}$ \\
\hline Total & 33 & 37 & 70 & Specificity $59.5 \%$. \\
\hline
\end{tabular}


to be a close correlation between the response in the TLP assay and that in the tuberculin skin test.

Furthermore, as shown in the table, the tests appeared equally capable of distinguishing between the patients with tuberculosis and those with other non-tuberculous disease. The skin test and the TLP test were compared with clinical, laboratory and radiological diagnosis of the disease - the criterion of validity being the diagnosis of the disease by physicians at the centres. All the basic measures of criterion validity (sensitivity, specificity and predictive value of a positive test) were similar for both tests and ranged between $58 \%$ and $64 \%$.

\section{Discussion}

The purpose of population screening is usually to detect as many cases as possible of the disease or risk factor that is being sought. The validity of a screening test is judged by determining its sensitivity, specificity and the predictive value of a positive test. ${ }^{6}$ These components are measured by reference to the results derived from a definitive diagnostic procedure. The predictive value of a positive test depends upon the prevalence of the disease or risk factor in the population tested, as well as the sensitivity and specificity of the test. The higher the prevalence, the more likely it is that a positive test will predict the disease.

In the present study, the sensitivity and specificity of the TLP test and the skin test were similar in the population tested, and both tests had predictive values of $c .60 \%$. Thus, although neither test had a high predictive value, the effectiveness of the TLP as a screening test for tuberculosis was similar to that of the tuberculin test.

Reports of the correlation between skin tests and lymphocyte proliferation assays in tuberculosis is not unanimous; in two studies, ${ }^{2,3}$ the correlation between the tests was statistically significant, whereas studies by others, ${ }^{8-10}$ demonstrated at best only a rough correlation. In this study, as in some of the previous ones, there was a highly significant association between the two tests as shown by the very high Odds ratio values. However, the higher correlation among nontuberculous subjects remains unexplained and further studies are needed to explore this finding. The discrepancies among the various studies cited may be accounted for by important differences, such as the number of subjects studied, whether or not patients with tuberculosis and normal tuberculin- or BCGpositive individuals were studied, and the nature of the antigens employed in the lymphocyte proliferation assay and the skin test.

It appears certain that the response to PPD in the skin test will remain the primary measure for screening populations for infection by $M$. tuberculosis, because of its low cost and the fact that it requires a minimum of equipment. However, the tuberculin test is difficult to standardise. The procedure is subject to variation in all its phases ;1 variability from reader to reader was found to be nearly as great as the biological variability from subject to subject. Also the use of the test requires that the individual be carefully identified and seen on a second occasion, usually $48 \mathrm{~h}$ after administration of the antigen. ${ }^{12,13}$ On the contrary, in the TLP test as described, only a small quantity of blood obtainable by fingerprick is required and subjects do not have to return for reading of results. Therefore, the simplified TLP test could have a place in the clinical armamentarium available for the diagnosis of tuberculosis. Future research leading to further simplification of this method may lead to it replacing intradermal skin testing.

We thank Dr S. Wartski, Head of the Pulmonary Diseases Unit of the Epidemiology Department of the Ministry of Health of Israel for his great help in the design of the study. We are also indebted to Drs J. H. Marcus and A. Hendler, Directors of the two centres in which the study was performed, for their generous assistance. We are very grateful to Professors L. Levy and C. Greenblatt for their useful criticism and suggestions in the preparation of the manuscript.

\section{References}

1. Collins FM. Immunology of tuberculosis. Am Rev Respir Dis $1982 ; 125: 42-49$.

2. Kerby GR. Correlation of tuberculin skin reaction with in vitro lymphocyte transformation. Am Rev Respir Dis 1968; 97 : 904-908.

3. Miller SD, Jones HE. Correlation of Lymphocyte transformation with tuberculin skin-test sensitivity. Am Rev Respir Dis 1973; 107: 530-538.

4. Cox RA, Lundberg DI, Arnold DR. Lymphocyte transformation assays as a diagnostic tool in tuberculosis of children. Am Rev Respir Dis 1981; 123: 627-630.

5. Frankenburg S. A simplified microtechnique for measuring human lymphocyte proliferation after stimulation with mitogen and specific antigen. J Immunol Methods 1988; 112: 177-182.

6. Abramson JH. Making sense of data: a self-instruction manual on the interpretation of epidemiological data. New York and Oxford, Oxford University Press. 1988: 136.

7. Cochrane AC, Holland WW. Validation of screening procedures. Br Med Bull 1971; 27 : 3-8.

8. Matsanoitis N, Tsenghi C, Economou-Marou C, Stavridaki CM. Skin hypersensitivity and in vitro lymphocyte reactivity to tuberculin in childhood. $J$ Pediatr 1968; 72 : 599-605.

9. McFarland W, Heilman DH. Comparison of lymphocyte transformation and intradermal reactions to tuberculins. Am Rev Respir Dis 1966; 93: 742-748.

10. Thomas JW, Naiman SC, Clements D. Lymphocyte transformation by phytohaemagglutinin II in the tuberculous patient. Can Med Assoc J 1967; 97: 836-840.

11. Bearman JE, Kleinman H, Glyer VV, La Croix OM. A study of variability in tuberculin test reading. Am Rev Respir Dis 1964; 90: 913-919.

12. Sokal JE. Measurement of delayed skin-test responses. $N$ Engl $J$ Med 1975; 293 : 501-502.

13. Snider DE. The tuberculin skin test. Am Rev Respir Dis 1982; 125: $108-118$. 